\title{
The Mediating Effect of Cognitive Appraisal on the Relationship Between Sleep Habits and the Stress Response Among Japanese Female College Students
}

\author{
Noriko Aizawa ( $\square$ aizawa.noriko@ocha.ac.jp ) \\ Institute for Education and Human Development, Ochanomizu University \\ Mika Omori \\ Faculty of Core Research Human Science Division, Ochanomizu University
}

\section{Research Article}

Keywords: cognitive appraisal, female college students, sleep habits, sleep irregularity, sleep pattern, stress response

Posted Date: January 15th, 2021

DOI: https://doi.org/10.21203/rs.3.rs-147428/v1

License: @) (1) This work is licensed under a Creative Commons Attribution 4.0 International License. Read Full License 


\section{Abstract}

Background: Undergraduate students tend to develop "evening-type" sleep patterns. However, recent research has reported that evening-type and irregular sleep habits are related to physical and mental stress responses, particularly in female students. Although the connection between sleep habits and the stress response has been well documented, the mechanism behind this relationship is currently unknown. Using the transactional model as a framework and female students as our target population, we examined whether sleep habits predict the stress response through the mediation of cognitive appraisals of one's own sleep habits.

Methods: Three hundred twenty-one Japanese female college students participated in this study. Participants completed measures of their sleep habits (sleep patterns and sleep irregularity), cognitive appraisals of their sleep habits (including four subscales: commitment, appraisal of influence, appraisal of threat, and controllability), stress responses (depression and anxiety), and control factors. The reliability and validity of the scales used in this study, except for sleep pattern and sleep irregularity, were confirmed in previous studies.

Results: Multiple-mediation-model analysis indicated that commitment mediates the relationship between sleep pattern and anxiety. Meanwhile, cognitive appraisals, as a whole, were found to have a mediating effect on the relationship between sleep irregularity and depression.

Conclusions: Our study revealed that cognitive appraisals mediated the relationship between sleep habits and the stress response. The findings also suggest that maintaining a low level of commitment might be effective for reducing anxiety, especially considering the difficulty associated with changing lifestyles. The findings of the present study should be useful for health education related to lifestyle.

\section{Background}

\section{Sleep habits and the stress response among college students}

In recent years, an increasing number of undergraduate students have begun to exhibit "evening-type" rhythms in their daily lives [1-4], referring to people who are more active from evening to night. The primary reasons that such students become evening-types and develop irregular sleep habits are related to the freedoms associated with college life, such as reductions in interference and constraints from their families and school, and greater freedom to make their own schedules [1]. One study of college students found that the average bedtime was 1:15 a.m. [2], with 74\% of female students reporting going to bed between 12:00 a.m. and 2:30 a.m. [5]. Based on these findings, it appears that most university students commence their sleeping phase after midnight, despite midnight being the most common bedtime for the general adult population in Japan [6]. Thus, the evening-type lifestyle seems to be a characteristic of Japanese undergraduate students $[3,4]$.

\section{Sleep habits and the stress response}

Several studies have reported that evening-types exhibit a stronger stress response than morning types [7]. It has also been reported that evening-type sleeping habits were related to poor physical and mental health conditions [8-12]. For example, studies of undergraduate students have found that evening-types had less efficient autonomic nervous system functioning in the morning [10], higher levels of psychological stress response [9], poorer physical and mental health [8], and lower academic performance [13] than morning types. Matsui et al. [14] found that university students with evening-type lifestyles reported relatively more fatigue and feelings of sickness, while Honda et al. [15] found that students with morning-type lifestyles showed a lower rate of such indefinite complaints. Thus, based on existing findings, it is becoming clear that evening-type and irregular sleep habits are associated with negative psychological and physical stress responses.

The impact of sleeping habits on the mind and body may be an especially important issue for female college students. For example, in a study by Kamimura et al. [9], $61 \%$ of female college students were evening-types. Moreover, compared to their male counterparts, female students took longer to fall asleep after going to bed, had poorer quality of sleep, and had worse 
overall sleeping conditions [2]. Many studies have reported that evening-type sleep habits were related to uncomfortable psychosomatic symptoms, particularly in young women. Examples include feelings of depression before the beginning of menstruation [16], irritability during menstruation [17], and menstrual pain [16, 18]. In addition, evening-type sleep habits were reported to be associated with various stress responses, such as awareness of fatigue $[9,18]$ and lack of concentration $[9,17]$. Thus, the relationship between women's sleep habits and various physical and psychological responses have been reported; however, the mechanism underlying the relationship between sleep habits and mental and physical health remain unclear and, consequently, further research is needed.

In the present study, we examined the effects of problematic sleep habits in female college students with a particular focus on depression and anxiety. This population was chosen as the target population because many studies have reported that psychological stress responses, such as depression and anxiety, were significantly more prevalent in women than in men [19, 20]. Thus, considering the above research literature, it is necessary to elucidate the process by which the sleep habits of female college students affect their physical and mental health. Therefore, in this study, we examined the influence of female college students' sleep habits on psychological stress (i.e., depression and anxiety), and also sought to determine the mechanism underlying the relationship between sleep habits and psychological stress.

\section{Important aspects of sleep habits}

Although there are many ways of measuring sleep, in the current study, we examined sleep habits by focusing on sleep pattern and sleep irregularity. Several previous studies have measured sleep habits in terms of "morningness-eveningness." Kleitman [21] defined "morningness-eveningness" as sleep-wake rhythms and sleep patterns, and many later studies adopted the latter term, "sleep pattern" [12, 22, 23]. In addition, several studies have recently suggested that lifestyle regularity, another concept related to sleep habits, has an important influence on health [1, 9, 13, 15, 22, 24]. For example, Honda et al. [15] showed that both an evening-type lifestyle and lifestyle irregularity were related to poor psychological health. Moreover, Takeda et al. [24] revealed that irregular lifestyles had a stronger influence on subjective symptoms than did sleep patterns. Considering the above, it seems clear that lifestyle irregularity is one of the most important aspects of sleep habits and may have an effect on the stress response.

\section{Sleep habits and cognitive appraisal}

We applied the transactional model $[25,26]$ to explain the influence of sleep habits on psychological stress responses. Lazarus and Folkman [26] emphasized the conducting of "cognitive appraisals" to determine the relationships between individuals and their environments. Exposure to environmental demands and pressures can cause people to feel stress, but there can be individual and group differences concerning the manner and degree of reaction to such stressors, as well as susceptibility and vulnerability to such events. Lazarus et al. [26] noted that individual differences regarding the responses people exhibit in specific stress situations had a strong influence on individuals' cognitive processes (i.e., cognitive appraisals) in relation to interpreting stressors [26]. Cognitive appraisal is defined as "the cognitive process of assessing how stressful the interaction between individuals and the environment is" [26] and includes the following: (1) the level of threat associated with the stressor (appraisal of threat), (2) how harmful the threat may be (appraisal of hazard), (3) how aggressively the individual intends to address the threat (appraisal of challenge), and (4) the degree to which the situation can be controlled (controllability). It has previously been clarified that cognitive appraisal strongly influences the selection of individual coping strategies and the expression of the stress response [27].

At the commencement of research investigating the effect of cognitive appraisal of sleep habits on health outcomes, Barber et al. [25] adopted Lazarus and Folkman's transactional model [26] as a framework and reported that cognitive appraisal had a mediating effect on the relationship between sleep habits and well-being. Further, Suzuki and Sakano [28] examined the relationship between cognitive appraisal and the stress response in major stress situations (task stress, interpersonal stress, physical threat stress), finding that the stress response became significant when the "appraisal of threat" of a stressful situation was high. It has also been reported that cognitive appraisal had a buffering effect on stress responses by contributing to coping ability. Furthermore, in interpersonal relationship stress situations, it was found that higher 
commitment (corresponding to the concept of "appraisal of challenge" [28]) was related to higher depression and anxiety. Cognitive appraisal is considered as having an important role in the mental health of individuals.

The relationship between sleep habits and the stress response, and between cognitive appraisal and the stress response have been clarified by previous studies [7-18, 25,28]. However, the degree to which cognitive appraisal affects the relationship between sleep habits and the stress response remains unclear. In order to obtain data that can be useful for improving the sleep habits and mental health of female college students, further investigation of the effects of sleep habits and cognitive appraisal on the stress response is needed. Based on the model of Barber et al. [25] and the findings of previous studies, we predicted that cognitive appraisals of sleep habits would mediate the relationship between poor sleep habits and heightened stress responses in female college students. In particular, we predicted that poor sleep habits would be related to (1) the perception that the individual must improve her lifestyle (commitment), (2) the perception that her life is threatened (threat), and (3) low controllability. Further, we predicted that these cognitive appraisals would exacerbate the stress response (i.e., depression and anxiety).

\section{Methods}

\section{Participants}

We recruited 321 Japanese female undergraduate and graduate students from a women's college in Tokyo as participants in the study. The study questionnaire was administered to 400 students, of whom 324 agreed to participate in the study (response rate $=81.0 \%$ ). The three respondents aged over 30 years old were presumed to have work experience, and their sleep habits and family composition were considered not representative of college students; therefore, these three students were excluded from the final analysis. Data from the remaining 321 participants were used in this study: 290 (90.3\%) were undergraduate students, 17 (5.3\%) were graduate students, and 14 (4.4\%) did not report their student status. The mean age was 19.72 years $(S D=1.73)$.

\section{Procedure}

Data were collected from October to December 2012. After the researchers obtained consent from course instructors, the questionnaire was distributed to the students in classrooms at the end of class. The students were informed that the survey was anonymous and that their participation was voluntary. Completion of the survey questionnaire was considered their indicating their consent to participate. Participants gave the completed questionnaire to the researchers in the class or dropped it into a collection box.

The present study was performed in accordance with the Declaration of Helsinki and was approved by the Ethics Committee of Ochanomizu University.

\section{Measures}

\section{Sleep habits}

Sleep habits were assessed using seven items developed for this study by referencing Ishihara et al. [29] and consisted of two subscales: Sleep Pattern and Sleep Irregularity. Sleep Pattern comprised five items measuring the tendency to engage in evening-type sleep habits and were selected from the Japanese version of the Morningness-Eveningness Questionnaire (MEQ; [30], Japanese version [29]). As many researchers have recommended the elimination of some items (e.g., those containing obsolete language, such as "night watch") for further investigations [22,31], we only selected items that were relevant to our aim of measuring sleep patterns (i.e., items that concern times during which respondents are active). The Cronbach's a score for the internal consistency reliability of the Sleep Pattern subscale was .65. Matsui (2010) noted that internal consistency is considered sufficient when the a coefficient is .60 or higher [32]; therefore, the reliability was considered sufficient. Examples of items included, "When you have no commitments the next day, at what time do you go to bed compared to your usual bedtime?" and "One hears about 'morning' and 'evening' types of people. Which ONE of these types do you consider yourself 
to be?" Items were responded to with a rating of 1 to 4 . Higher scores on the Sleep Pattern subscale reflect a greater tendency to have an evening-type sleep pattern.Two items were used to measure sleep irregularity, which were specially created for this study: for example, "Do you think you have a regular sleep rhythm on weekdays?" (a reverse-scored item). The Cronbach's a score for the internal consistency reliability of the Sleep Irregularity subscale was .82. Participants responded to the items using a four-point scale ( 1 = "strongly disagree" to 4 = "strongly agree"). Higher scores reflect a greater tendency to engage in irregular sleeping patterns.

\section{Cognitive appraisals of sleep habits}

Cognitive appraisals of sleep habits were assessed using eight items from the Cognitive Appraisal Rating Scale (CARS) [28]. The CARS includes four subscales that assess commitment (appraisal of the extent to which one actively commits to the situation), appraisal of influence, appraisal of threat, and controllability. The CARS has demonstrated sufficient validity and reliability [28]. In order to measure cognitive appraisals of sleep habits, items that featured the phrase "this situation" were altered to state "my present sleep habit" or "my present sleep habits" (e.g., "I think my present sleep habit is important to me"). Participants rated each item using a four-point scale ( 1 = "strongly disagree" to 4 = "strongly agree"). Regarding internal consistency reliability, the Cronbach's a scores of the CARS subscales were as follows: commitment .78, appraisal of influence .82 , appraisal of threat .93, and controllability .56. For each cognitive appraisal component, a higher score reflects a greater tendency to use that type of cognitive appraisal.

\section{Stress response}

Stress response was measured using two subscales from the Stress Self-Rating Scale (SSRS) [33]: Depression (five items) and Anxiety (five items). Participants rated each item using a four-point scale ( 1 = "strongly disagree" to $4=$ "strongly agree"). Support for the validity and internal consistency reliability of the SSRS was confirmed in a previous study [34]. The Cronbach's a scores for the Depression and Anxiety subscales were .88, and .85, respectively. Higher scores indicate a stronger stress response in terms of anxiety and depression.

\section{Control variables}

In the analysis, it was necessary to control for the effects of confounding variables, such as the perceived impact of stressors and dispositional optimism. Previous research has reported that the higher the score for a perceived stressor, the stronger the stress response, and the higher the score for dispositional optimism, the weaker the stress response [22, 35, 36]. To include these variables as control variables, they were measured in the questionnaire. Perceived impact of a stressor was measured using the 35-item scale concerning the daily stressors of graduate students included in the SSRS [33]. Following the recommendations of Fukui and Fukui [22], we removed the scale measuring degree of distress ( $0=$ no stressor, $1=$ "none at all" to 4 = "very much"), retaining only the scale concerning whether the stressor had been experienced $(0=$ "no," $1=$ "experienced"). Thus, in the present study, higher scores reflected a greater number of stressors experienced. Dispositional optimism was measured using the Japanese version of the Life Orientation Test-Revised (LOT-R) [37], which is a measure of generalized positive and negative outcome expectancies [35].

\section{Results}

\section{Preliminary analyses}

Bivariate correlations and descriptive statistics, including the means and standard deviations of the variables and the reliability coefficients of the scales, are shown in Table 1.

Table 1. Bivariate correlation matrix for variables 


\begin{tabular}{|c|c|c|c|c|c|c|c|c|c|c|c|c|c|c|c|c|c|c|c|c|c|}
\hline & & $\bar{M}$ & SD & $\alpha$ & 1 & & 2 & & 3 & & 4 & & 5 & & 6 & & 7 & & 8 & 9 & \\
\hline 1 & Stressor & .55 & .19 & - & & & & & & & & & & & & & & & & & \\
\hline 2 & Optimism & 2.63 & .47 & .72 & -.24 & ** & & & & & & & & & & & & & & & \\
\hline 3 & Sleep Pattern & 2.50 & .57 & .65 & -.10 & & .05 & & & & & & & & & & & & & & \\
\hline 4 & $\begin{array}{l}\text { Sleep } \\
\text { Irregularity }\end{array}$ & 2.40 & .82 & .82 & .03 & & -.03 & & .38 & ** & & & & & & & & & & & \\
\hline 5 & Commitment & 2.58 & .73 & .78 & .21 & ** & -.04 & & .28 & ** & .47 & $* *$ & & & & & & & & & \\
\hline 6 & $\begin{array}{l}\text { Appraisal of } \\
\text { Influence }\end{array}$ & 3.55 & .53 & .82 & .07 & & .03 & & -.03 & & .00 & & .14 & $* *$ & & & & & & & \\
\hline 7 & $\begin{array}{l}\text { Appraisal of } \\
\text { Threat }\end{array}$ & 2.41 & .90 & .93 & .12 & * & -.16 & $* *$ & .27 & ** & .48 & ** & .62 & $* *$ & .12 & * & & & & & \\
\hline 8 & Controllability & 2.72 & .60 & .56 & -.08 & & .18 & $* *$ & -.30 & ** & -.43 & $* *$ & -.26 & $* *$ & .12 & * & -.31 & $* *$ & & & \\
\hline 9 & Depression & 2.16 & .85 & .88 & .35 & ** & -.38 & $* *$ & .12 & * & .13 & * & .26 & $* *$ & .12 & * & .30 & $* *$ & -.13 & * & \\
\hline & Anxiety & 2.13 & .77 & .85 & .31 & ** & -.16 & $* *$ & .08 & & .07 & & .25 & $* *$ & .06 & & .10 & & -.08 & .73 & ** \\
\hline
\end{tabular}

${ }^{*} p<.05,{ }^{* *} p<.01$; for stressor, "no" is coded as " 0 " and "experienced" is coded as " 1. ."

Evening-type sleep pattern was found to be positively associated with commitment $(r=.28, p<.01)$ and appraisal of threat $(r$ $=.27, p<.01)$, but negatively associated with controllability $(r=-.30, p<.01)$. Further, sleep irregularity was also found to be significantly associated with higher commitment $(r=.47, p<.01)$, higher appraisal of threat $(r=.48, p<.01)$, and lower controllability $(r=-.43, p<.01)$. In the correlation analyses, sleep pattern and irregularity were found to have weak positive association with depression but no associations with appraisal of influence or anxiety.

The control variables were found to be significantly associated with stress responses. Specifically, stressors were positively related to depression $(r=.35, p<.01)$ and anxiety $(r=.31, p<.01)$, whereas dispositional optimism was negatively related to depression $(r=-.38, p<.01)$ and anxiety $(r=-.16, p<.01)$.

\section{Testing multiple mediation models}

We hypothesized that sleep habits would predict stress responses, such as depression and anxiety, through cognitive appraisals. Consequently, four multiple mediation models (two for each sleep habit and two for each stress response) were created using Preacher and Hayes' (2008) procedure (available through SPSS macro) [38], which also allowed for covariates. This approach, using bootstrap analysis, enabled us to explore mediating relationships, thereby overcoming the limitations of previous methods (assuming normal distribution, or if it is impossible to test the indirect effect; for a review, see Preacher and Hayes 2008) [38]. Indirect effects were tested using a bootstrapping procedure, which involved running 5,000 samples with $95 \%$ confidence intervals (Cls).

\section{Sleep pattern and the stress response}

Table 2 presents the direct and indirect effects of the proposed mediators on the relationship between sleep pattern and the stress response. Stressors and optimism were added as covariates. Fig. 1 depicts the hypothesized model.

Table 2. Results of the multiple mediation model for sleep pattern regarding depression and anxiety. 


\begin{tabular}{|c|c|c|c|c|c|c|c|}
\hline & \multicolumn{3}{|c|}{ Depression } & \multicolumn{4}{|c|}{ Anxiety } \\
\hline & $b$ & $S E$ & $t$ value & $b$ & $S E$ & \multicolumn{2}{|c|}{$t$ value } \\
\hline \multicolumn{8}{|c|}{ Partial effects of covariates on stress response } \\
\hline Stressor & 1.19 & .24 & $4.86 * *$ & 1.04 & .24 & 4.35 & $* *$ \\
\hline Optimism & -.58 & .10 & $-5.89 * *$ & -.21 & .10 & -2.21 & * \\
\hline \multicolumn{8}{|c|}{ Effects of sleep pattern on mediators } \\
\hline Commitment & .34 & .07 & $5.30 * *$ & .34 & .07 & 5.30 & $* *$ \\
\hline Appraisal of Influence & .00 & .05 & -.10 & .00 & .05 & -.10 & \\
\hline Appraisal of Threat & .39 & .08 & $4.85 * *$ & .39 & .08 & 4.85 & $* *$ \\
\hline Controllability & -.33 & .05 & $-6.17 * *$ & -.33 & .05 & -6.17 & $* *$ \\
\hline \multicolumn{8}{|c|}{ Direct mediating effects on stress response } \\
\hline Commitment & .09 & .08 & 1.12 & .27 & .08 & 3.55 & $* *$ \\
\hline Appraisal of Influence & .13 & .09 & 1.48 & .06 & .08 & .70 & \\
\hline Appraisal of Threat & .11 & .06 & 1.75 & -.15 & .06 & -2.44 & * \\
\hline Controllability & .05 & .08 & .61 & -.01 & .08 & -.17 & \\
\hline \multicolumn{8}{|c|}{ Effects of sleep pattern on stress response } \\
\hline Total & .23 & .07 & $3.13 * *$ & .14 & .07 & 1.97 & $*$ \\
\hline Direct & .17 & .08 & $2.15 *$ & .10 & .08 & 1.31 & \\
\hline Indirect effects & $b$ & $S E$ & $95 \%$ BCa $C I$ & $b$ & $S E$ & $95 \%$ BCa CI & \\
\hline Total & .06 & .04 & $(-.02 ; .13)$ & .04 & .03 & $(-.03 ; .11)$ & \\
\hline Commitment & .03 & .03 & $(-.02 ; .08)$ & .09 & .03 & $(.04 ; .16)$ & \\
\hline Appraisal of Influence & .00 & .01 & $(-.02 ; .01)$ & .00 & .00 & $(-.01 ; .01)$ & \\
\hline Appraisal of Threat & .04 & .03 & $(-.00 ; .10)$ & -.06 & .03 & $(-.12 ;-.01)$ & \\
\hline Controllability & -.02 & .03 & $(-.08 ; .04)$ & .00 & .03 & $(-.05 ; .06)$ & \\
\hline \multirow[t]{2}{*}{ Model statistics } & $R$ & $R^{2}$ & $F_{(7,279)}$ & $R$ & $R^{2}$ & \multicolumn{2}{|l|}{$F_{(7,279)}$} \\
\hline & .53 & .29 & 15.91 & .40 & .16 & 7.59 & $* *$ \\
\hline
\end{tabular}

${ }^{*} p<.05,{ }^{* *} p<.01$.

Regarding the relationship between sleep pattern and depression, sleep pattern (a greater tendency to have an evening-type sleep pattern) positively predicted depression $(b=.23, p<.01)$. With regard to cognitive appraisal, sleep pattern positively predicted commitment $(b=.34, p<.01)$ and appraisal of threat $(b=.39, p<.01)$, and negatively predicted controllability $(b=$ $-.33, p<.01)$. There was no significant relationship to appraisal of influence. The direct effects of cognitive appraisal types on depression were not significant. The direct effect of sleep pattern on depression was reduced with the inclusion of the mediators and covariates $(b=.17, p<.05)$. The indirect effect of cognitive appraisals, both cumulatively and individually, showed that cognitive appraisals did not contribute to the prediction of depression (i.e., the $95 \% \mathrm{Cl}$ included zero).

In the results for the multiple mediation model for anxiety, sleep pattern positively predicted anxiety $(b=.14, p<.01)$. Sleep pattern also positively predicted commitment $(b=.34, p<.01)$ and appraisal of threat $(b=.39, p<.01)$, whereas sleep pattern negatively predicted controllability $(b=-.33, p<.01)$. There was no significant relationship to appraisal of influence. In addition, commitment and appraisal of threat significantly positively predicted anxiety. The direct effect of sleep pattern on anxiety became nonsignificant with the inclusion of the mediators $(b=.10)$. Although the total effect was not significant $(b=$ $.04,95 \% \mathrm{Cl}=-.03$ to .11$)$, commitment $(b=.09,95 \% \mathrm{Cl}=.04$ to .16$)$, and appraisal of threat $(b=-.06,95 \% \mathrm{Cl}=-.12$ to -.01$)$ contributed uniquely to the mediation effect (i.e., the $95 \% \mathrm{Cl}$ did not include zero). However, because appraisal of threat did not correlate with anxiety, we could not interpret the indirect effect of threat.

\section{Sleep irregularity and the stress response}

Table 3 shows the direct and indirect effects of the proposed mediators in the relationship between sleep irregularity and the stress response. Stressors and optimism were added as covariates. Fig. 2 depicts the hypothesized model.

Table 3. Results of the multiple mediation model for sleep irregularity regarding depression and anxiety. 


\begin{tabular}{|c|c|c|c|c|c|c|c|}
\hline & \multicolumn{3}{|c|}{ Depression } & \multicolumn{4}{|c|}{ Anxiety } \\
\hline & $b$ & $S E$ & t value & $b$ & $S E$ & \multicolumn{2}{|c|}{$t$ value } \\
\hline \multicolumn{8}{|c|}{ Partial effects of covariates on stress response } \\
\hline Stressor & 1.07 & .24 & $4.39 * *$ & .96 & .24 & $4.07 *$ & $* *$ \\
\hline Optimism & -.56 & .10 & $-5.61 * *$ & -.20 & .10 & $-2.06 *$ & * \\
\hline \multicolumn{8}{|c|}{ Effects of sleep irregularity on mediators } \\
\hline Commitment & .38 & .05 & $8.17 * *$ & .38 & .05 & $8.17 *$ & $* *$ \\
\hline Appraisal of Influence & .02 & .04 & .51 & .02 & .04 & .51 & \\
\hline Appraisal of Threat & .48 & .06 & $8.58 * *$ & .48 & .06 & $8.58 *$ & ** \\
\hline Controllability & -.28 & .04 & $-7.30 * *$ & -.28 & .04 & $-7.30 *$ & $* *$ \\
\hline \multicolumn{8}{|c|}{ Direct mediating effects on stress response } \\
\hline Commitment & .10 & .08 & 1.28 & .29 & .08 & $3.66 *$ & $* *$ \\
\hline Appraisal of Influence & .13 & .09 & 1.54 & .06 & .08 & .75 & \\
\hline Appraisal of Threat & .12 & .07 & 1.76 & -.15 & .06 & $-2.26 *$ & \\
\hline Controllability & .00 & .08 & .03 & -.04 & .08 & -.50 & \\
\hline \multicolumn{8}{|c|}{ Effects of sleep irregularity on stress response } \\
\hline Total & .12 & .05 & $2.24 *$ & .06 & .05 & 1.10 & \\
\hline Direct & .03 & .07 & .40 & .01 & .06 & .12 & \\
\hline Indirect effects & $b$ & $S E$ & $95 \%$ BCa $C I$ & $b$ & $S E$ & $95 \%$ BCа CI & \\
\hline Total & .10 & .04 & $(.02 ; .18)$ & .05 & .04 & $(-.02 ; .13)$ & \\
\hline Commitment & .04 & .03 & $(-.02 ; .10)$ & .11 & .03 & $(.05 ; .18)$ & \\
\hline Appraisal of Influence & .00 & .01 & $(-.01 ; .02)$ & .00 & .00 & $(-.01 ; .01)$ & \\
\hline Appraisal of Threat & .06 & .03 & $(-.00 ; .12)$ & -.07 & .03 & $(-.14 ;-.01)$ & \\
\hline Controllability & .00 & .03 & $(-.05 ; .05)$ & .01 & .03 & $(-.04 ; .07)$ & \\
\hline \multirow[t]{2}{*}{ Model statistics } & $R$ & $R^{2}$ & $F_{(7,279)}$ & $R$ & $R^{2}$ & \multicolumn{2}{|l|}{$F_{(7,279)}$} \\
\hline & .52 & .27 & 14.51 & .39 & .15 & $7.15^{*}$ & \\
\hline
\end{tabular}

${ }^{*} p<.05,{ }^{* *} p<.01$

Sleep irregularity positively predicted depression $(b=.12, p<.05)$, commitment $(b=.38, p<.01)$, and appraisal of threat $(b=$ $.48, p<.01)$, and negatively predicted controllability $(b=-.28, p<.01)$. There was no significant relationship to appraisal of influence. The direct effects of the cognitive appraisal types on depression were not significant, and the direct effect of sleep irregularity on depression became nonsignificant with the inclusion of the mediators and covariates $(b=.03)$. Analysis of the indirect effect $(b=.10,95 \% \mathrm{Cl}=.02$ to -.18$)$ showed that cognitive appraisals as a group contributed to the prediction of depression. However, no individual type of cognitive appraisal showed a unique mediating effect (i.e., the $95 \% \mathrm{Cl}$ included zero).

For the mediation model for sleep irregularity and anxiety, sleep irregularity did not predict anxiety $(b=.06)$. This was despite the fact that there were significant relationships between sleep irregularity and cognitive appraisals and between cognitive appraisals and anxiety, as well as indirect effects. Because we could not confirm the preconditional relation between sleep irregularity and anxiety, we could not interpret the mediation effects.

\section{Discussion}

In this study, we proposed that based on the model of Barber et al. [25], cognitive appraisals of sleep habits (commitment, appraisal of influence, appraisal of threat, and controllability) would mediate the relationship between sleep habits and stress responses (anxiety and depression). The results of a multiple-mediation-model analysis indicated that cognitive appraisals mediated the relationship between sleep habits and stress responses in a different pattern in the sleep-pattern model and sleep-irregularity model. In the sleep-pattern model, an evening-type sleep pattern was found to be associated with greater depression and anxiety, as well as greater commitment, greater appraisal of threat, and less controllability. Moreover, commitment was associated with greater anxiety, and the mediating effect of commitment was confirmed. This is consistent with past research concerning interpersonal stressors [28]. Only depression was affected by the evening-type sleep pattern. 
Being awake late at night might directly exacerbate depressive mood. In the sleep-irregularity model, sleep irregularity was found to be associated with greater depression, greater commitment, greater appraisal for threat, and less controllability. Meanwhile, no individual type of cognitive appraisal affected depression; however, the cumulative indirect effect of the four types of cognitive appraisal was confirmed. Sleep irregularity was associated with greater commitment, greater threat, and less controllability, and these cognitive appraisals were related to greater depression. These findings suggest that assessing one's desire to manage evening-type sleep contributes to anxiety, and thinking multifacetedly about the effects of sleep irregularities contributes to depression.

This study builds on past research by exploring the mediating effect of cognitive appraisal on the relationship between sleep habits and the stress response in female college students. Supporting our hypotheses, appraisal of commitment was found to mediate the relationship between sleep pattern and anxiety, and cognitive appraisals, as a whole, were found to mediate the relationship between sleep irregularity and depression, even when controlling for stressors and optimism. Therefore, the relationship between sleep pattern and anxiety, and between sleep irregularity and depression, can be fully explained by cognitive appraisals, including their significant indirect effects.

These findings have practical implications in that educational institutions can use them when providing sleep-health education for college students. As mentioned earlier, because the sleep habits of university or college students are becoming irregular and trending toward an evening-type lifestyle, it is important for educational institutions to hold seminars that provide health education for students concerning the dangers of this lifestyle. Needless to say, such health education, which could inform adolescents of the importance of sufficient sleep and the benefits of morningness, might promote students' awareness regarding their lifestyles. On the other hand, from our research, it was suggested that awareness of commitments, such as "I should go to bed before 22:00" or "I have to sleep more than eight hours," can occasionally have a bad influence on mental health. In other words, when lifestyle change is hindered by existing restrictions (e.g., needing to be awake late at night for a part-time job or to meet friends, or needing to wake up early to attend class), a high awareness of commitment (e.g., I should be living a healthy lifestyle) is likely to increase frustration and anxiety. In such situations, managing or changing how the student cognitively appraises the situation may be an effective way to minimize or alleviate anxiety as a stress response. Therefore, sleep-health education should include a framework of stress appraisals and inform individuals of not only the importance of sleep habits but also the effect of cognitive appraisals. For example, if an individual's sleep-related lifestyle is difficult to change, it might be more effective for the student to stop searching for a way to improve their sleep habits and avoid trying to drastically change their lifestyle.

\section{Sleep habits and their effect on stressful situations}

In the present study, the participants reported feeling that their sleep habits needed improvement, were threatening to their health, and were uncontrollable. In addition, the results indicated that higher commitment to changing one's sleep habits was related to a stress response, which is consistent with the findings of previous research (cf. Suzuki and Sakano, 1998); high commitment was related to high stress responses in interpersonal relationships (as a stressful situation) [28]. For individuals with evening-type or irregular sleep habits, there is often no clear cause that can be addressed (interpersonal relationship stress as well); therefore, their desire to change but uncertainty regarding the appropriate approach leads to the stress response, and this can have a negative effect on mental health.

\section{Limitations and future research}

There are several limitations to this study. First, all the participants in this study were female students. In order to generalize the findings of the present study to male students and non-students, research including men and other populations (e.g., different age groups and countries, working adults) is needed. Second, the subscale assessing controllability had low internal consistency reliability. Further studies are needed to validate the effect of controllability by using other measures of the construct or evaluating its reliability with other populations. Third, it is possible that some of the study participants had sleep disorders. Therefore, it is necessary to account for such disorders and eliminate those who cannot be labeled as morning- or evening-types. 
Despite the above limitations, our results have significance, as we revealed the importance of cognitive appraisals of sleep habits, especially when students have evening-type or irregular lifestyles. More specifically, we observed how people appraise situations or lifestyles that can play an important role not only in stress-coping contexts [26] but also in sleep-habit contexts [25]. Conducting research concerning cognitive appraisals and sleep habits may lead to the creation of effective healthpromoting education for college and university students who are in the process of developing their own lifestyles. In the future, to corroborate and disclose the importance of cognitive appraisal of sleep habits, studies targeting people who have low controllability regarding their sleep habits (e.g., night-shift workers) are needed.

\section{Conclusions}

In conclusion, cognitive appraisal of sleep habits, especially appraisal of commitment, may be a pathway through which evening-type and irregular sleep habits affect greater stress responses (depression and anxiety). In particular, increased commitment, a greater sense that one's lifestyle is threatening to their health, and a lack of controllability may be outcomes of evening-type and irregular sleep habits, which subsequently exacerbate the stress response. In addition, the findings suggest that avoiding thinking about or assessing one's commitment to improving one's lifestyle to change their sleep habits has the potential to suppress or alleviate the stress response.

Because our study targeted healthy female college students, we believe that the findings can be used for effective health education, promoting the role of cognitive appraisal. To promote physical health, it is desirable to implement education that propagates the benefits or importance of morningness and sufficient sleep.

However, emphasizing regimented sleep habits can sometimes have harmful effects. Our research revealed that even though it is difficult to improve sleeping habits by oneself, trying to improve habits through certain cognitive appraisals can be stressful. In such cases, avoiding being overly conscious of the need to improve one's lifestyle is the key to suppressing the stress response. The findings of the present study should be useful for health education related to lifestyle.

\section{List Of Abbreviations}

CARS - Cognitive Appraisal Rating Scale

$\mathrm{Cl}$ - Confidence interval

LOT-R - Life Orientation Test-Revised

MEQ - Morningness-Eveningness Questionnaire

SSRS - Stress Self-Rating Scale

\section{Declarations}

\section{Ethics approval and consent to participate}

This study was approved by the Ochanomizu University Research Ethics Committee (ref. 24-22). All participants received a clear explanation of the research aim, their right to privacy, the anonymity of their responses, and their right to refuse to participate or cease participation at any time without penalty. Completion of the survey was considered to indicate consent.

\section{Consent for publication}

Not applicable.

\section{Availability of data and materials}


The dataset used and analyzed during the current study is available from the corresponding author on reasonable request.

\section{Competing interests}

The authors declare that they have no competing interests

\section{Funding}

Not applicable.

\section{Authors' contributions}

NA designed the study, conducted the data analyses, interpreted the data, drafted the manuscript, and revised the manuscript. MO contributed to the study design, supervised data analysis and interpretation, and contributed to the drafting and revising of the manuscript. All authors approved the final version of the manuscript.

\section{Acknowledgments}

Not applicable.

\section{References}

1. Takeuchi T, Inugami M, Ishihara K, Fukuda K. Construction of sleep-hygiene scales and classification of sleep patterns in undergraduates. The Japanese Journal of Educational Psychology. 2000;48(3):294-304 (in Japanese).

2. Kurokawa T, Ishimura I. Effect of sleep conditions on truancy in university students. Bulletin of Clinical Psychology, Tokyo Seitoku University. 2013;13:3-16 (in Japanese).

3. Ohta Y, Saeki K, Ohta K. A study on the lifestyle and the health consciousness of male \& female students. Bulletin of Fukuoka University of Education, Part 5; Art, Health and Physical Education, and Home Economics. 1994(43):83-99 (in Japanese).

4. Tokuda K. A preliminary study of life style and mental health in university students: On life style, resilience and sleep. Ritsumeikan Journal of Human Sciences. 2013;27:91-100 (in Japanese).

5. Honda T, Sakamoto A, Hara E. On the actual state of lifestyle of female university students: An analysis by the intensity level of physical activities on daily life. Fukuoka Jogakuin University Bulletin. 2006;7:15-21 (in Japanese).

6. NHK Broadcasting Culture Research Institute. Sleeping time keeps decreasing, male housework time is increasing: From the 2010 NHK Japanese time use survey. 2 https://www.nhk.or.jp/bunken/english/reports/pdf/report_110401.pdf Accessed 21 Dec 2018.

7. Hori, T. Suimin Shinrigaku. Kyoto: Kitaohji shobo; 2008 (in Japanese).

8. Harada T, Kadowaki A, Shinomiya $H$, Takeuchi $H$. Relationship between watching late night TV and morningnesseveningness of 18-22-year old Japanese students. Sleep Biol Rhythms. 2004;2(1):97-98.

9. Kamimura Y, Takeda N, Sakuma A, Teraoka C, Kishida N. Effect of night type life style on psychological stress response and dietary habit of women university students. Bulletin of Faculty of Human Life and Environmental Science Hiroshima Prefectural Women's University. 2000;6:11-19 (in Japanese).

10. Yamaguchi M, Watanabe T, Takagi A, Wakisaka S, Sakane N, Moritani T, et al. Morningness-eveningness preference and the autonomic nervous system activity in the morning among female university students. Journal of Japanese Society of Psychosomatic Obstetrics and Gynecology. 2011;16(2):160-168 (in Japanese).

11. Mecacci L, Rocchetti G. Morning and evening types: Stress-related personality aspects. Pers Individ Diff. 1998;25(3):537542.

12. Willis TA, O'Connor DB, Smith L. The influence of morningness-eveningness on anxiety and cardiovascular responses to stress. Physiol Behav. 2005;85(2):125-133. 
13. Kiuchi A, Nakamura T, Arai H, Urai R, Hashimoto K. Relationship between lifestyle and the acquired number of academic credits in first-year college students. Japanese Association of University Physical Education and Sports. 2010;7:69-76 (in Japanese).

14. Matsui T, Furumi K, Tsunoda T, Matsumoto K, Teruya K, Tamura H, et al. A study on the health administration of students: The relationship between personal health practices and morning-evening type. Journal of the Kyorin Medical Society. 1989;20(4):447-454 (in Japanese).

15. Honda M, Suzuki S, Shirota Y, Kaneko S, Takahashi S. A study on perceived health of morningness-eveningness type and subjective health. Japanese Journal of Health and Human Ecology. 1994;60(5):266-273 (in Japanese).

16. Takeuchi H., Oishi T, Harada T. Association between morningness-eveningness preference and mental/physical premenstrual symptoms in Japanese females 12 to 31 years of age. Chronobiol Int. 2005;22:1055-1068.

17. Nakade M, Takeuchi $\mathrm{H}$, Kurotani $\mathrm{M}$, Harada T. Effects of meal habits and alcohol/cigarette consumption on morningnesseveningness preference and sleep habits by Japanese female students aged 18-29. J Physiol Anthropol. 2009;28:83-90.

18. Negriff S, Dorn LD. Morningness/evenigness and menstrual symptoms in adolescent females. J Psychosom Res. 2009;67:169-172.

19. Suzuki S, Shimada H, Miura M, Katayanagi K, Umano R, Sakano Y. Development of a new psychological stress response scale (SRS-18) and investigation of the reliability and the validity. Japanese Journal of Behavioral Medicine. 1997;4(1):22-29 (in Japanese).

20. Zender R, Olshansky E. Women's mental health: Depression and anxiety. Nurs Clin North Am. 2009;44(3):355-364.

21. Kleitman N. Sleep and wakefulness. Chicago: University of Chicago Press; 1963.

22. Fukui Y, Fukui T. Cognitive appraisal of sleep habits influences stress reactions among university students. The Japanese Journal of Health Psychology. 2009;22(2):52-59 (in Japanese).

23. Roeser K, Meule A, Schwerdtle B, Kübler A, Schlarb AA. Subjective sleep quality exclusively mediates the relationship between morningness-eveningness preference and self-perceived stress response. Chronobiol Int. 2012;29(7):955-960.

24. Takeda N, Kamimura Y, Teraoka C, Moriwaki H, Sakuma A, lida T, et al. Relationship between life and diet of women university students by night type life and subjective symptoms. Bulletin of Faculty of Human Life and Environmental Science, Hiroshima Women's University. 2001;7:17-30 (in Japanese).

25. Barber LK, Rupprecht EA, Munz DC. Sleep habits may undermine well-being through the stressor appraisal process. J Happiness Stud. 2014;15(2):285-299.

26. Lazarus RS, Folkman S. Stress, appraisal, and coping. New York: Springer; 1984.

27. Folkman S, Lazarus, RS. Cognitive theories of stress and the issue of circularity. In: Appley MH, Trumbull R, editors. Dynamics of stress: Physiological, psychological, and social perspectives. New York: Plenum Press; 1986. p. 63-80.

28. Suzuki S, Sakano Y. Development of a cognitive appraisal rating scale (CARS) and its validation. Waseda Human Science Research. 1998;7:113-124 (in Japanese).

29. Ishihara K, Miyashita A, Inugami M, Fukuda K, Yamazaki K, Miyata Y. The results of investigation by the Japanese version of Morningness-Eveningness Questionnaire. Shinrigaku kenkyu [The Japanese Journal of Psychology]. 1986;57(2):87-91 (in Japanese).

30. Horne JA, Ostberg O. A self-assessment questionnaire to determine morningness-eveningness in human circadian rhythms. Int J Chronobiol. 1976;4(2):97-110.

31. Honda M, Suzuki S, Ube H, Shirota Y, Kaneko S, Fujima K, et al. Construct validity of the Japanese version of Horne and Ostberg's Morningness-Eveningness Questionnaire in women college students. Japanese Journal of Health and Human Ecology. 1995;61(3):139-149 (in Japanese).

32. Matsui Y. Shinrigaku ronbun no kakikata. Tokyo: Kawade Shobo Shinsya; 2010 (in Japanese).

33. Ozeki Y, Haraguchi M, Tsuda A. A covariance structural analysis to the psychological stress process in university students. The Japanese Journal of Health Psychology. 1994;7(2):20-36 (in Japanese).

Page $12 / 13$ 
34. Ozeki Y. Refining the stress self-rating scale for university students - Toward a transactional analysis. The Annual of the Graduate School of Comparative Studies of International Cultures and Societies. 1993;1:95-114 (in Japanese).

35. Scheier MF, Carver CS, Bridges MW. Distinguishing optimism from neuroticism (and trait anxiety, self-mastery, and selfesteem): A reevaluation of the Life Orientation Test. J Pers Soc Psychol. 1994;67(6):1063-1078.

36. Scheier MF, Carver CS. On the power of positive thinking: The benefits of being optimistic. Curr Dir Psychol Sci. 1993;2(1):26-30.

37. Sakamoto S, Tanaka E. A study of the Japanese version of revised Life Orientation Test. The Japanese Journal of Health Psychology. 2002;15(1):59-63 (in Japanese).

38. Preacher KJ, Hayes AF. Asymptotic and resampling strategies for assessing and comparing indirect effects in multiple mediator models. Behav Res Methods. 2008;40(3):879-891.

\section{Figures}

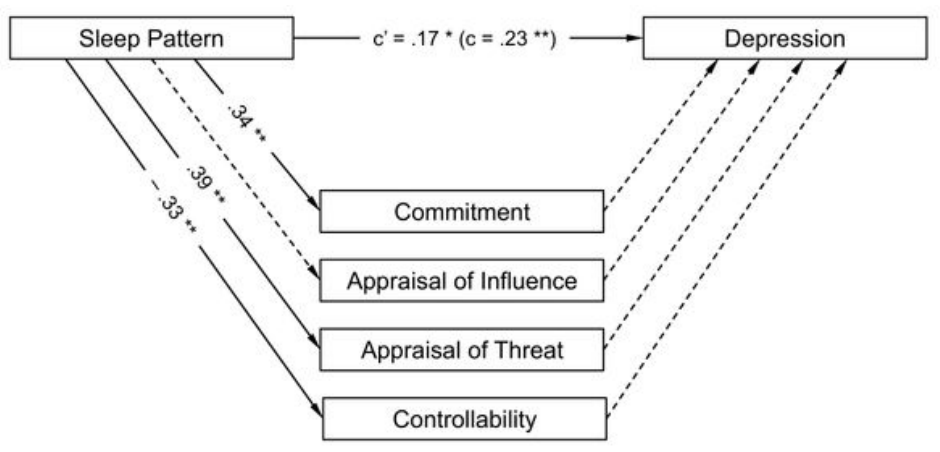

Model A.1. Effects of sleep pattern on depression through cognitive appraisals.

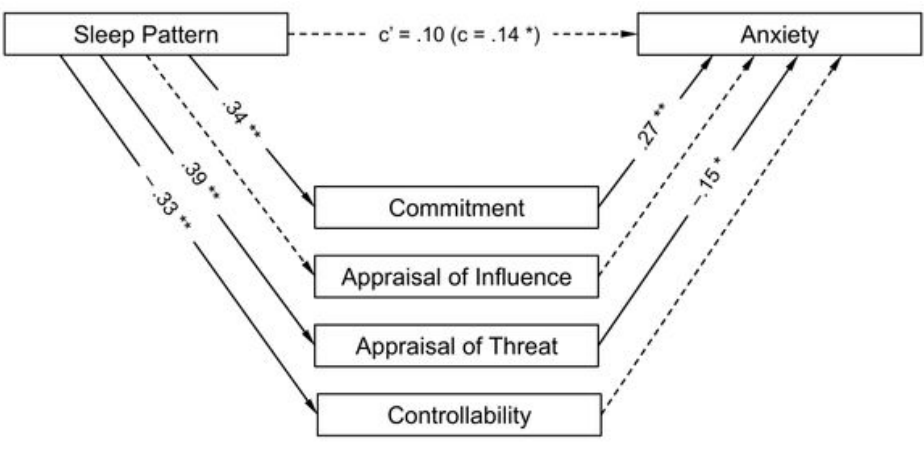

Model A.2. Effects of sleep pattern on anxiety through cognitive appraisals.

\section{Figure 1}

Effects of sleep pattern on stress responses through cognitive appraisals. Analyses control for stressor and optimism. ${ }^{*} \mathrm{p}$ $.05, * * p<.01$.

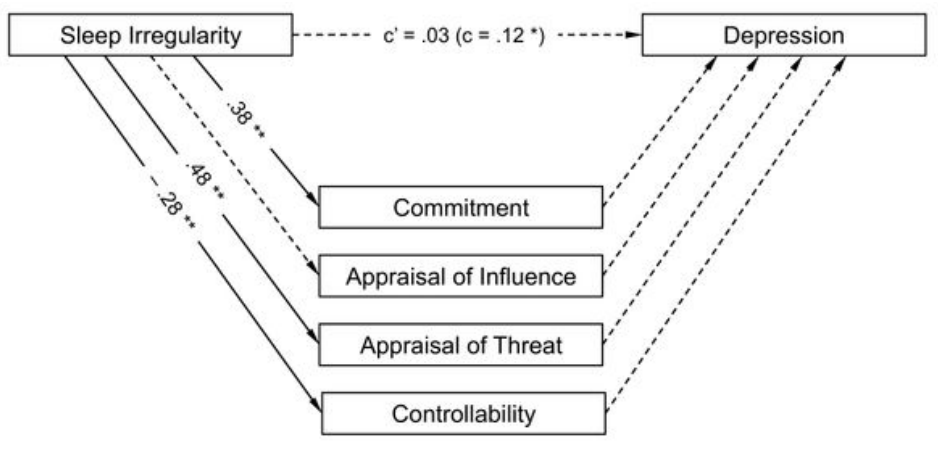

Model B.1. Effects of sleep irregularity on depression through cognitive appraisals.

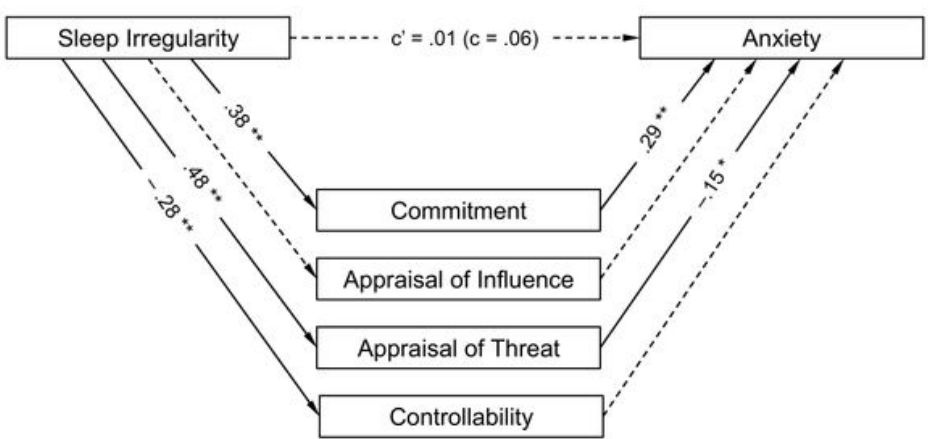

Model B.2. Effects of sleep irregularity on anxiety through cognitiveappraisals.

\section{Figure 2}

Effects of sleep irregularity on stress responses through cognitive appraisals. Analyses control for stressor and optimism. ${ }^{\star} \mathrm{p}<$ $.05, * * p<.01$. 